Kragujevac Journal of Mathematics

Volume 45(4) (2021), Pages 635-645.

\title{
THE MAXIMUM NORM ANALYSIS OF SCHWARZ METHOD FOR ELLIPTIC QUASI-VARIATIONAL INEQUALITIES
}

\author{
MOHAMMED BEGGAS ${ }^{1}$ AND MOHAMMED HAIOUR ${ }^{2}$
}

\begin{abstract}
In this paper, we present a maximum norm analysis of an overlapping Schwartz method on non matching grids for a quasi-variational inequality, where the obstacle and the second member depend on the solution. Our result improves and generalizes some previous results.
\end{abstract}

\section{INTRODUCTION}

Historically, Schwarz method has been introduced by Herman Amondus Schawarz, in order to resolve a purely theoretical matters. The Schawarz alternating method has been used to solve the stationary or evolutionary boundary valued problems, on domain which consists of two or more overlapping sub-domains, see for example [6,7]. The solution is approximated by an infinite sequence of function, the result which is the resolution of a sequence of stationary or evolutionary boundary valued problems, in each of sub-domain.

In this work, we are interested in the analysis of error estimates in uniform norm for the quasi-variational inequality. Our goal is to generalize and improve some previous results given in $[2-4,10,11]$ which concerning analysis of error estimates in uniform norm for the elliptic quasi-variational inequality. As in [2] they got the following approximation:

$$
\left\|u_{i}-u_{i h}^{n+1}\right\|_{\infty} \leq C h^{2}|\log h|^{3}, \quad i=1,2,
$$

Key words and phrases. Schwarz method, quasi-variational inequalities, weakly subsequenti ally continuous, $L^{\infty}$-error estimates.

2010 Mathematics Subject Classification. Primary: 05C38. Secondary: 15A15, 05A15, $15 \mathrm{~A} 18$.

DOI 10.46793/KgJMat2104.635B

Received: February 08, 2018.

Accepted: April 08, 2019. 
for the problem

$$
\left\{\begin{array}{l}
a(u, v-u) \geq(f, v-u) \text { in } \Omega, \quad \text { for all } v \in K \\
u \leq \psi, \quad v \leq \psi
\end{array}\right.
$$

where $K$ is a convex, closed and not empty set. In [4], they have obtained the same approximation for the following problem:

$$
\left\{\begin{array}{l}
a(u, v-u) \geq(f(u), v-u) \text { in } \Omega, \quad \text { for all } v \in K(u) \\
u \leq \psi, \quad v \leq \psi
\end{array}\right.
$$

also, for the non-coercive variational inequality, it has been reached in [11], the same approximation mentioned above. In [10], the authors studied a quasi-variational inequality related to control ergodic problem

$$
\left\{\begin{array}{l}
b\left(u_{\alpha}, v-u_{\alpha}\right) \geq\left(f+r u_{\alpha}, v-u_{\alpha}\right), \quad \alpha \in(0,1), \\
u_{\alpha} \leq M u_{\alpha}, \quad v \leq M u_{\alpha}
\end{array}\right.
$$

and they got the following result:

$$
\left\|u_{\alpha_{i}}-u_{\alpha_{i} h}^{n+1}\right\|_{\infty} \leq C \alpha^{-2} h^{2}|\log h|^{4}, \quad i=1,2 .
$$

Finally in [3], the authors studied the following problem:

$$
\left\{\begin{array}{l}
a(u, v-u) \geq(f, v-u), \quad \text { for all } v \in K, \\
u \leq M u, \quad M u \geq 0, \\
M u=k+\inf _{\varepsilon \geq 0, x+\varepsilon \in \bar{\Omega}} u(x+\varepsilon), \\
\frac{\partial u}{\partial \eta}=\varphi \text { in } \Gamma_{0} \text { and } u=0 \text { in } \Gamma / \Gamma_{0},
\end{array}\right.
$$

and they obtained the following result:

$$
\left\|u_{i}-u_{i h}^{n+1}\right\|_{\infty} \leq C h^{2}|\log h|^{3}, \quad i=1,2 .
$$

For our work, we claim about the general problem where the second member and the obstacle are related to the solution

$$
\left\{\begin{array}{l}
a(u, v-u) \geq(f(u), v-u) \text { in } \Omega, \quad \text { for all } v \in K_{g}(u), \\
u \leq M u, v \leq M u \\
u=g \text { on } \partial \Omega
\end{array}\right.
$$

The outline of the paper, is as follows: in the second section, we will mention the same notations and assumptions, in the third section we will give our continuous problem, analogously in section four, we will define the discrete problem. Section five, is devoted to the $L^{\infty}$-error analysis of the method. 


\section{Notation And Assumptions}

Let $\Omega$ be an open in $\mathbb{R}^{n}$, with sufficiently smooth boundary $\partial \Omega$. For $u, v \in H^{1}(\Omega)$, consider the bilinear form as follows:

$$
a(u, v)=\int_{\Omega}\left(\sum_{1 \leq i, j \leq n} a_{i j}(x) \frac{\partial u}{\partial x_{i}} \frac{\partial v}{\partial x_{j}}+\sum_{1 \leq i \leq n} a_{i}(x) \frac{\partial u}{\partial x_{i}} v+a_{0}(x) u . v\right) d x
$$

where $a_{i j}(x), a_{i}(x), a_{0}(x), x \in \bar{\Omega}, 1 \leq i, j \leq n$, are sufficiently smooth coefficients and satisfying the following conditions:

$$
\begin{gathered}
\sum_{1 \leq i, j \leq n} a_{i j} \xi_{i} \xi_{j} \geq \nu|\xi|^{2}, \quad \xi \in \mathbb{R}^{n}, \nu>0 \\
a_{0}(x) \geq \beta>0
\end{gathered}
$$

where $\beta$ is a constant. The operator $M$ is given by $M u=k+\inf _{\varepsilon \geq 0, x+\varepsilon \in \bar{\Omega}} u(x+\varepsilon)$, where $k>0$ and $M$ satisfies

$$
M u \in W^{2, \infty}(\Omega), \quad M u \geq 0 \text { on } \partial \Omega: 0 \leq g \leq M u,
$$

where $g$ is a regular function defined on $\partial \Omega$. Let $f$ be a Lipschitzian non decreasing nonlinear function with rate $\alpha$ satisfying $\frac{\alpha}{\beta}<1$ and $f \in L^{\infty}(\Omega)$, and $K_{g}(u)$ is an implicit convex and non empty set which defined as follows:

$$
K_{g}(u)=\left\{v \in H^{1}(\Omega), v=g \text { on } \partial \Omega, v \leq M u \text { in } \Omega\right\} .
$$

\section{The Continuous Problem}

We consider the following problem: Find $u \in k_{g}(u)$ the solution of

$$
\left\{\begin{array}{l}
a(u, v-u) \geq(f(u), v-u) \text { in } \Omega, \quad \text { for all } v \in K_{g}(u), \\
u \leq M u, \quad v \leq M u \\
u=g \text { on } \partial \Omega
\end{array}\right.
$$

We will present some results for our problem as the existence, uniqueness and other optimal properties which given in previous papers where we need them in the sequel.

Theorem 3.1 ([5]). Under the previous conditions the problem (3.1) has an unique solution $u \in K_{g}(u)$. Moreover, we have

$$
u \in W^{2, p}(\Omega), \quad 2 \leq p \leq \infty .
$$

Lemma 3.1 ([6]). For all $u$ and $\tilde{u} \in K_{g}(u)$, we have

(a) if $u \leq \tilde{u}$, then $M u \leq M \tilde{u}$ and $M(u+\lambda)=M(u)+\lambda$ for all $\lambda \in \mathbb{R}$;

(b) $\|M u-M \tilde{u}\|_{L^{\infty}(\Omega)} \leq\|u-\tilde{u}\|_{L^{\infty}(\Omega)}$. 
3.1. The continuous Schwarz sequences. We decompose $\Omega$ in two sub-domains $\Omega_{1}, \Omega_{2}$ such that $\Omega=\Omega_{1} \cup \Omega_{2}$ and $u$ satisfies the local regularity condition:

$$
u / \Omega_{i} \in W^{2, p}\left(\Omega_{i}\right), \quad i=1,2, \text { and } 2 \leq p<\infty,
$$

denote by $\partial \Omega_{i}$ the boundary of $\Omega_{i}$ and $\Gamma_{1}=\partial \Omega_{1} \cap \Omega_{2}, \Gamma_{2}=\partial \Omega_{2} \cap \Omega_{1}, \Gamma_{1} \cap \Gamma_{2}=\emptyset$.

We define the following process. Choose $u_{0}=k$ to be given, and define the alternating Schwarz sequences $\left(u_{1}^{n+1}\right)$ on $\Omega_{1}$ such that $u_{1}^{n+1} \in K\left(u_{1}^{n}\right)$ is solution of the following problem:

$$
\left\{\begin{array}{l}
a_{1}\left(u_{1}^{n+1}, v-u_{1}^{n+1}\right) \geq\left(f_{1}\left(u_{1}^{n}\right), v-u_{1}^{n+1}\right), \\
u_{1}^{n+1} \leq M u_{1}^{n}, \\
u_{1}^{n+1}=u_{2}^{n} \text { on } \Gamma_{1}, \quad v=u_{2}^{n} \text { on } \Gamma_{1}
\end{array}\right.
$$

and $\left(u_{2}^{n+1}\right)$ on $\Omega_{2}$ such that $u_{2}^{n+1} \in K\left(u_{2}^{n}\right)$ solution of the following problem:

$$
\left\{\begin{array}{l}
a_{2}\left(u_{2}^{n+1}, v-u_{2}^{n+1}\right) \geq\left(f_{2}\left(u_{2}^{n}\right), v-u_{2}^{n+1}\right), \\
u_{2}^{n+1} \leq M u_{2}^{n}, \\
u_{2}^{n+1}=u_{1}^{n} \text { on } \Gamma_{2}, \quad v=u_{1}^{n} \text { on } \Gamma_{2},
\end{array}\right.
$$

where $f_{i}=f / \Omega_{i}, i=1,2$, and $\left(a_{i}(u, v)\right.$ the form bilinear which defined in (2).

\subsection{Geometrical convergence.}

Theorem 3.2 ([3]). The sequences $\left(u_{1}^{n+1}\right),\left(u_{2}^{n+1}\right), n \geq 0$, produced by the Schawarz alternating method converge geometrically to the solution $u$ of the problem (3.1), more precisely, there exist two constants $K_{1}, K_{2} \in(0,1)$ such that for all $n \geq 0$, we have

$$
\begin{aligned}
& \left\|u_{1}-u_{1}^{n+1}\right\|_{L^{\infty}\left(\Omega_{1}\right)} \leq K_{1}^{n} K_{2}^{n}\left\|u^{0}-u\right\|_{L^{\infty}\left(\Gamma_{1}\right)}, \\
& \left\|u_{2}-u_{2}^{n+1}\right\|_{L^{\infty}\left(\Omega_{2}\right)} \leq K_{1}^{n+1} K_{2}^{n}\left\|u^{0}-u\right\|_{L^{\infty}\left(\Gamma_{2}\right)} .
\end{aligned}
$$

We will show an important proposition, which give the continuous dependence to the second member, the data $g$ and the obstacle. We note that $u=\sigma(f(u), M u, g)$, $\tilde{u}=\sigma(f(\tilde{u}), M \tilde{u}, \tilde{g})$, where $u, \tilde{u} \in K_{g}(u)$.

Proposition 3.1. Under the previous hypotheses and notations, we have

$$
\|u-\tilde{u}\|_{L^{\infty}\left(\Omega_{i}\right)} \leq\|f(u)-f(\tilde{u})\|_{L^{\infty}\left(\Omega_{i}\right)}+\|M u-M \tilde{u}\|_{L^{\infty}\left(\Omega_{i}\right)}+\|g-\tilde{g}\|_{L^{\infty}\left(\Gamma_{i}\right)},
$$

where $\Gamma_{i}=\partial \Omega_{i} \cap \Omega_{j}, i, j=1,2$, and $i \neq j$.

Proof. Setting

$$
\Phi=\|f(u)-f(\tilde{u})\|_{L^{\infty}\left(\Omega_{i}\right)}+\|M u-M \tilde{u}\|_{L^{\infty}\left(\Omega_{i}\right)}+\|g-\tilde{g}\|_{L^{\infty}\left(\Gamma_{i}\right)},
$$

we have

$$
f(u) \leq f(\tilde{u})+f(u)-f(\tilde{u}) \leq f(\tilde{u})+\|f(u)-f(\tilde{u})\| \leq f(\tilde{u})+\Phi .
$$

Similarly, we have $g \leq \tilde{g}+\Phi$ and $M u \leq M \tilde{u}+\phi$. 
Now, making use of Lemma 3.2, we obtain

$$
\begin{aligned}
\sigma(f(u), M u, g) & \leq \sigma(f(\tilde{u})+\Phi, M \tilde{u}+\Phi, \tilde{g}+\Phi) \\
& \leq(f(\tilde{u}), M \tilde{u}, \tilde{g})+\Phi
\end{aligned}
$$

so, $\sigma(f(u), M u, g)-\sigma(f(\tilde{u}), M \tilde{u}, \tilde{g}) \leq \Phi$. Since $(f(u), M u, g)$ and $(f(\tilde{u}), M \tilde{u}, \tilde{g})$ are symmetrical, we have $\sigma(f(\tilde{u}), M \tilde{u}, \tilde{g})-\sigma(f(u), M u, g) \leq \Phi$, and then

$$
\|u-\tilde{u}\|_{L^{\infty}\left(\Omega_{i}\right)} \leq\|f(u)-f(\tilde{u})\|_{L^{\infty}\left(\Omega_{i}\right)}+\|M u-M \tilde{u}\|_{L^{\infty}\left(\Omega_{i}\right)}+\|g-\tilde{g}\|_{L^{\infty}\left(\Gamma_{i}\right)} .
$$

Remark 3.1. If $M u=M \tilde{u}$, we have

$$
\|u-\tilde{u}\|_{L^{\infty}\left(\Omega_{i}\right)} \leq\|f(u)-f(\tilde{u})\|_{L^{\infty}\left(\Omega_{i}\right)}+\|g-\tilde{g}\|_{L^{\infty}\left(\Gamma_{i}\right)} .
$$

\section{The Discrete Problem}

We denote by $V_{h}$ the standard piecewise linear finite element space, we consider the discrete quasi-variational inequality. Find $u_{h} \in K_{g h}\left(u_{h}\right)$ such that:

$$
\left\{\begin{array}{l}
a\left(u_{h}, v-u_{h}\right) \geq\left(f\left(u_{h}\right), v-u_{h}\right), \quad \text { for all } u_{h}, v \in K_{g h}\left(u_{h}\right), \\
u_{h} \leq r_{h} M u_{h} \\
u_{h}=\pi_{h} g \text { on } \partial \Omega
\end{array}\right.
$$

where $f \in L^{\infty}(\Omega) ; M u_{h}=k+\inf _{\varepsilon \geq 0, x+\varepsilon \in \bar{\Omega}} u_{h}(x+\varepsilon)$ and

$$
K_{g h}\left(u_{h}\right)=\left\{v \in V_{h}: v=\pi_{h} g \text { on } \partial \Omega, v \leq r_{h} M u_{h} \text { in } \Omega\right\} .
$$

We denote $\pi_{h}$ the interpolation operator on $\partial \Omega$ and $r_{h}$ is the usual finite element restriction operator in $\Omega$.

4.1. The discrete maximum principle. We assume that the respective matrices resulting from the discretization of problems (3.2), (3.1) are $M$-matrice [9].

Theorem 4.1 ([1]). Let $u$ and $u_{h}$ be the solutions of problem (3.1) and (4.1) respectively, there exists a constant $C_{1}$ independent of $h$ such that

$$
\left\|u-u_{h}\right\|_{L^{\infty}(\Omega)} \leq C_{1} h^{2} \log |h|^{2} .
$$

Similarly, for the continuous case we will establish the discrete version of the lemma.

Lemma 4.1. For all $u_{h}$ and $\tilde{u_{h}} \in K_{g}\left(u_{h}\right)$ we have

(a) if $u_{h} \leq \tilde{u_{h}}$, then $M u_{h} \leq M \tilde{u_{h}}$ and $M\left(u_{h}+\lambda\right)=M\left(u_{h}\right)+\lambda$ for all $\lambda \in \mathbb{R}$;

(b) $\left\|M u_{h}-M \tilde{u}_{h}\right\|_{L^{\infty}(\Omega)} \leq\left\|u_{h}-\tilde{u_{h}}\right\|_{L^{\infty}(\Omega)}$. 
4.2. The discrete Schwarz sequences. For $i=1,2$, let $V_{h_{i}}=V_{h}\left(\Omega_{i}\right)$ be the space of continuous picewise linear function on $\tau_{h_{i}}$, which vanish on $\partial \Omega \cap \partial \Omega_{i}$. For $w \in C\left(\Gamma_{i}\right)$, we define

$$
V_{h_{i}}^{(w)}=\left\{v \in V_{h_{i}}, v=0 \text { on } \partial \Omega_{i} \cap \partial \Omega, v=\pi_{h_{i}}(w) \text { on } \Gamma_{2}\right\}
$$

where $\tau_{h_{i}}$ be a standard regular finite element triangulation in $\Omega_{i}, h_{i}$ being the mesh size. We suppose that the two triangulation are mutually independent on $\Omega_{1}, \Omega_{2}$, a triangle belonging to one triangulation does not necessarily belong to the other.

We now define the discrete countreparts of the continuous Schwarz sequences defined in (3.2) and (3.1) respectively, by $\left(u_{1 h}^{n+1}\right) \in V_{h_{1}}^{\left(u_{2 h}^{n}\right)}$, where $\left(u_{1 h}^{n+1}\right)$ is the solution of

$$
\left\{\begin{array}{l}
a_{1}\left(u_{1 h}^{n+1}, v-u_{1 h}^{n+1}\right) \geq\left(f_{1}\left(u_{1 h}^{n}\right), v-u_{1 h}^{n+1}\right), \quad \text { for all } v \in V_{h_{1}}^{\left(u_{2 h}^{n}\right)} \\
u_{1 h}^{n+1} \leq r_{h} M u_{1 h}^{n}, \quad v \leq r_{h} m u_{1 h}^{n} \\
u_{1 h}^{n+1}=u_{2 h}^{n} \text { on } \Gamma_{1}, \quad v=u_{2 h}^{n} \text { on } \Gamma_{1}
\end{array}\right.
$$

and $\left(u_{2 h}^{n+1}\right) \in V_{h 2}^{\left(u_{1 h}^{n+1}\right)}$ such that $\left(u_{2 h}^{n+1}\right)$ is the solution of

$$
\left\{\begin{array}{l}
a_{2}\left(u_{2 h}^{n+1}, v-u_{2 h}^{n+1}\right) \geq\left(f_{2}\left(u_{2 h}^{n}\right), v-u_{2 h}^{n+1}\right), \quad \text { for all } v \in V_{h_{2}}^{\left(u_{1 h}^{n}\right)}, \\
u_{2 h}^{n+1} \leq r_{h} M u_{2 h}^{n}, \quad v \leq r_{h} M u_{2 h}^{n} \\
u_{2 h}^{n+1}=u_{1 h}^{n} \text { on } \Gamma_{2}, \quad v=u_{2 h}^{n} \text { on } \Gamma_{2} .
\end{array}\right.
$$

We will finish this section by the discrete version of Proposition 3.1, this version plays an important role in the sequel.

Proposition 4.1. Using the notations

$$
\begin{aligned}
& u_{h}=\sigma\left(f\left(u_{h}\right), M u_{h}, \pi_{h} g\right), \\
& \tilde{u}_{h}=\sigma_{h}\left(f\left(\tilde{u_{h}}, M \tilde{u_{h}}, \pi_{h} \tilde{g}\right),\right.
\end{aligned}
$$

where $u_{h}, \tilde{u_{h}} \in K_{g}\left(u_{h}\right)$, we have

$\left\|u_{h}-\tilde{u_{h}}\right\|_{L^{\infty}\left(\Omega_{i}\right)} \leq\left\|f\left(u_{h}\right)-f\left(\tilde{u_{h}}\right)\right\|_{L^{\infty}\left(\Omega_{i}\right)}+\left\|M u_{h}-M \tilde{u_{h}}\right\|_{L^{\infty}\left(\Omega_{i}\right)}+\left\|\pi_{h} g-\pi_{h} \tilde{g}\right\|_{L^{\infty}\left(\Gamma_{i}\right)}$,

$\Gamma_{i}=\partial \Omega_{i} \cap \Omega_{j}, i, j=1,2$, and $i \neq j$.

Proof. Similar for the continuous case.

Remark 4.1. If $M u_{h}=M \tilde{u_{h}}$, we obtain

$$
\left\|u_{h}-\tilde{u_{h}}\right\|_{L^{\infty}\left(\Omega_{i}\right)} \leq\left\|f\left(u_{h}\right)-f\left(\tilde{u_{h}}\right)\right\|_{L^{\infty}\left(\Omega_{i}\right)}+\left\|\pi_{h} g-\pi_{h} \tilde{g}\right\|_{L^{\infty}\left(\Gamma_{i}\right)} .
$$

\section{5. $L^{\infty}$-ERror Estimate}

We will use the algorithmic approach, which was used in [2,4], but our problem is more complicated because the second member and the obstacle are related to the solution. 
5.1. Auxiliary sequences. We introduce two discrete auxiliary sequences. Starting from $w_{i h}^{0}=u_{i h}^{0}=r_{h} M u_{h}^{0}=k, i=1,2$, define the sequences $\left(w_{1 h}^{n+1}\right)$ such that $w_{1 h}^{n+1} \in V_{h_{1}}^{u_{2}^{n}}$

$$
\left\{\begin{array}{l}
a_{1}\left(w_{1 h}^{n+1}, v-w_{1 h}^{n+1}\right) \geq\left(f_{1}\left(u_{1 h}^{n}\right), v-w_{1 h}^{n+1}\right), \quad \text { for all } v \in V_{h_{1}}^{\left(u_{2}^{n}\right)}, \\
w_{1 h}^{n+1} \leq r_{h} M u_{1 h}^{n}, \quad v \leq r_{h} M u_{1 h}^{n},
\end{array}\right.
$$

and $\left(w_{2 h}^{n+1}\right)$ such that $w_{2 h}^{n+1} \in V_{h_{2}}^{\left(u_{1}^{n+1}\right)}$ is a solution of

$$
\left\{\begin{array}{l}
a_{2}\left(w_{2 h}^{n+1}, v-w_{2 h}^{n+1}\right) \geq\left(f_{2}\left(\begin{array}{l}
n \\
2 h
\end{array}\right), v-w_{2 h}^{n+1}\right), \quad \text { for all } v \in V_{h_{2}}^{\left(u_{1}^{n+1}\right)} \\
w_{2 h}^{n+1} \leq r_{h} M u_{2 h}^{n}, \quad v \leq r_{h} M u_{2 h}^{n}
\end{array}\right.
$$

Note that $w_{i h}^{n+1}$ is the finite element approximation of $u_{i}^{n+1}$ which defined in (3.2) and (3.1). The following lemma will play a crucial role in proving the main result of this paper. The demonstration of the lemma is an adaptation of the one in [2], given for the problem of variational inequality.

Lemma 5.1. We have the following inequalities:

$$
\begin{aligned}
& \left\|u_{1}^{n+1}-u_{1 h}^{n+1}\right\|_{1} \leq \sum_{p=1}^{n+1}\left\|u_{1}^{p}-w_{1 h}^{p}\right\|_{1}+\sum_{p=0}^{n+1}\left\|u_{2}^{p}-w_{2 h}^{p}\right\|_{2}, \\
& \left\|u_{2}^{n+1}-u_{2 h}^{n+1}\right\|_{2} \leq \sum_{p=0}^{n+1}\left\|u_{2}^{p}-w_{2 h}^{p}\right\|_{2}+\sum_{p=1}^{n+1}\left\|u_{1}^{p}-w_{1 h}^{p}\right\|_{1} .
\end{aligned}
$$

Proof. In order to simplify the notation, we will adopt the following notations:

$$
\begin{aligned}
|\cdot|_{1} & =\|\cdot\|_{L^{\infty}\left(\Gamma_{1}\right)}, \quad|\cdot|_{2}=\|\cdot\|_{L^{\infty}\left(\Gamma_{2}\right)}, \\
\|\cdot\|_{1} & =\|\cdot\|_{L^{\infty}\left(\Omega_{1}\right)}, \quad\|\cdot\|_{2}=\|\cdot\|_{L^{\infty}\left(\Omega_{2}\right)}, \\
\pi_{h_{1}} & =\pi_{h_{2}}=\pi_{h}, \quad h_{1}=h_{2}=h .
\end{aligned}
$$

Started for $n=0$, using the Remark 4.1, we get

$$
\begin{aligned}
\left\|u_{1}^{1}-u_{1 h}^{1}\right\|_{1} & \leq\left\|u_{1}^{1}-w_{1 h}^{1}\right\|_{1}+\left\|w_{1 h}^{1}-u_{1 h}^{1}\right\|_{1} \\
& \leq\left\|u_{1}^{1}-w_{1 h}^{1}\right\|_{1}+\left\|f_{1}\left(u_{1}^{0}\right)-f_{1}\left(u_{1 h}^{0}\right)\right\|_{1}+\left|\pi_{h} M u_{2}^{0}-\pi_{h} M u_{2 h}^{0}\right|_{1} \\
& \leq\left\|u_{1}^{1}-w_{1 h}^{1}\right\|_{1}+\left|M u_{2}^{0}-M u_{2 h}^{0}\right|_{1} \\
\left\|u_{1}^{1}-u_{1 h}^{1}\right\|_{1} & \leq\left\|u_{1}^{1}-w_{1 h}^{1}\right\|_{1}+\left\|M u_{2}^{0}-M u_{2 h}^{0}\right\|_{2}
\end{aligned}
$$

and, from Lemma 4.1, we obtain

$$
\left\|u_{1}^{1}-u_{1 h}^{1}\right\|_{1} \leq\left\|u_{1}^{1}-w_{1 h}^{1}\right\|_{1}+\left\|u_{2}^{0}-u_{2 h}^{0}\right\|_{2} .
$$


Similarly, we obtain

$$
\begin{aligned}
\left\|u_{2}^{1}-u_{2 h}^{1}\right\|_{2} & \leq\left\|u_{2}^{1}-w_{2 h}^{1}\right\|_{2}+\left\|w_{2 h}^{1}-u_{2 h}^{1}\right\|_{2} \\
& \leq\left\|u_{2}^{1}-w_{2 h}^{1}\right\|_{2}+\left\|f_{2}\left(u_{2 h}^{0}\right)-f_{2}\left(u_{2 h}^{0}\right)\right\|_{2}+\left|\pi_{h} M u_{1}^{1}-\pi_{h} M u_{1 h}^{1}\right|_{2} \\
& \leq\left\|u_{2}^{1}-w_{2 h}^{1}\right\|_{2}+\left|M u_{1}^{1}-M u_{1 h}^{1}\right|_{2} \\
& \leq\left\|u_{2}^{1}-w_{2 h}^{1}\right\|_{2}+\left\|M u_{1}^{1}-M u_{1 h}^{1}\right\|_{1}
\end{aligned}
$$

and

From (5.3), we get

$$
\left\|u_{2}^{1}-u_{2 h}^{1}\right\|_{2} \leq\left\|u_{2}^{1}-w_{2 h}^{1}\right\|_{2}+\left\|u_{1}^{1}-u_{1 h}^{1}\right\|_{1} .
$$

$$
\left\|u_{2}^{1}-u_{2 h}^{1}\right\|_{2} \leq\left\|u_{1}^{1}-w_{1 h}^{1}\right\|_{1}+\left\|u_{2}^{0}-u_{2 h}^{0}\right\|_{2}+\left\|u_{2}^{1}-w_{2 h}^{1}\right\|_{2},
$$

so

$$
\begin{aligned}
& \left\|u_{1}^{1}-u_{1 h}^{1}\right\|_{1} \leq \sum_{p=1}^{1}\left\|u_{1}^{p}-w_{1 h}^{p}\right\|_{1}+\sum_{p=0}^{0}\left\|u_{2}^{0}-u_{2 h}^{0}\right\|_{2}, \\
& \left\|u_{2}^{1}-u_{2 h}^{1}\right\|_{2} \leq \sum_{p=0}^{1}\left\|u_{2}^{p}-w_{2 h}^{p}\right\|_{2}+\sum_{p=1}^{1}\left\|u_{1}^{p}-w_{1 h}^{p}\right\|_{1} .
\end{aligned}
$$

For $n=1$, we have

$$
\begin{aligned}
\left\|u_{1}^{2}-u_{1 h}^{2}\right\|_{1} & \leq\left\|u_{1}^{2}-w_{1 h}^{2}\right\|_{1}+\left\|w_{1 h}^{2}-u_{1 h}^{2}\right\|_{1} \\
& \leq\left\|u_{1}^{2}-w_{1 h}^{2}\right\|_{1}+\left\|f\left(u_{1 h}^{1}\right)-f\left(u_{1 h}^{1}\right)\right\|_{1}+\left|\pi_{h} M u_{2}^{1}-\pi_{h} M u_{2 h}^{1}\right|_{1}, \\
& \leq\left\|u_{1}^{2}-w_{1 h}^{2}\right\|_{1}+\left|M u_{2}^{1}-M u_{2 h}^{1}\right|_{1} \\
& \leq\left\|u_{1}^{2}-w_{1 h}^{2}\right\|_{1}+\left\|u_{2}^{1}-u_{2 h}^{1}\right\|_{2} .
\end{aligned}
$$

From (5.4), we get

$$
\left\|u_{1}^{2}-u_{1 h}^{2}\right\|_{1} \leq\left\|u_{2}^{1}-w_{1 h}^{2}\right\|_{1}+\left\|u_{2}^{1}-w_{2 h}^{1}\right\|_{2}+\left\|u_{1}^{1}-w_{1 h}^{1}\right\|_{1}+\left\|u_{2}^{0}-u_{2 h}^{0}\right\|_{2} .
$$

Similarly, we obtain

$$
\begin{aligned}
\left\|u_{2}^{2}-u_{2 h}^{2}\right\|_{2} & \leq\left\|u_{2}^{2}-w_{2 h}^{2}\right\|_{2}+\left\|w_{2 h}^{2}-u_{2 h}^{2}\right\|_{2} \\
& \leq\left\|u_{2}^{2}-w_{2 h}^{2}\right\|_{2}+\left\|f\left(u_{2 h}^{2}\right)-f\left(u_{2 h}^{2}\right)\right\|_{2}+\left|\pi_{h} M u_{1}^{2}-\pi_{h} M u_{1 h}^{2}\right|_{2} \\
& \leq\left\|u_{2}^{2}-w_{2 h}^{2}\right\|_{2}+\left\|u_{1}^{2}-u_{1 h}^{2}\right\|_{1} .
\end{aligned}
$$

From (5.5), we get

$\left\|u_{2}^{2}-u_{2 h}^{2}\right\|_{2} \leq\left\|u_{2}^{2}-w_{2 h}^{2}\right\|_{2}+\left\|u_{2}^{1}-w_{1 h}^{2}\right\|_{1}+\left\|u_{2}^{1}-w_{2 h}^{1}\right\|_{2}+\left\|u_{1}^{1}-w_{1 h}^{1}\right\|_{1}+\left\|u_{2}^{0}-u_{2 h}^{0}\right\|_{2}$,

where

$$
\left\|u_{1}^{2}-u_{1 h}^{2}\right\|_{1} \leq \sum_{p=1}^{2}\left\|u_{1}^{p}-w_{1 h}^{p}\right\|_{1}+\sum_{p=0}^{1}\left\|u_{2}^{p}-w_{2 h}^{p}\right\|_{2}
$$

and

$$
\left\|u_{2}^{2}-u_{2 h}^{2}\right\|_{2} \leq \sum_{p=0}^{2}\left\|u_{1}^{p}-w_{2 h}^{p}\right\|_{2}+\sum_{p=1}^{2}\left\|u_{1}^{p}-w_{1 h}^{p}\right\|_{1} .
$$


We go to the second step. Suppose that

$$
\left\|u_{2}^{n}-u_{2 h}^{n}\right\|_{2} \leq \sum_{p=0}^{n}\left\|u_{2}^{p}-w_{2 h}^{p}\right\|_{2}+\sum_{p=1}^{n}\left\|u_{1}^{p}-w_{1 h}^{p}\right\|_{1} .
$$

We claim the first inequality, for $i=1$,

$$
\begin{aligned}
\left\|u_{1}^{n+1}-u_{1 h}^{n+1}\right\|_{1} & \leq\left\|u_{1}^{n+1}-w_{1 h}^{n+1}\right\|_{1}+\left\|w_{1 h}^{n+1}-u_{1 h}^{n+1}\right\|_{1} \\
& \leq\left\|u_{1}^{n+1}-w_{1 h}^{n+1}\right\|_{1}+\left\|f_{1}\left(u_{1 h}^{n}\right)-f_{1}\left(u_{1 h}^{n}\right)\right\|_{1}+\left|\pi_{h} M u_{2}^{n}-\pi_{h} M u_{2 h}^{n}\right|_{1} \\
& \leq\left\|u_{1}^{n+1}-w_{1 h}^{n+1}\right\|_{1}+\left\|M u_{2}^{n}-M u_{2 h}^{n}\right\|_{2} \\
& \leq\left\|u_{1}^{n+1}-w_{1 h}^{n+1}\right\|_{1}+\left\|u_{2}^{n}-u_{2 h}^{n}\right\|_{2} .
\end{aligned}
$$

From (5.6), we get

$$
\left\|u_{1}^{n+1}-u_{1 h}^{n+1}\right\|_{1} \leq\left\|u_{1}^{n+1}-w_{1 h}^{n+1}\right\|_{1}+\sum_{p=0}^{n}\left\|u_{1}^{p}-w_{1 h}^{p}\right\|_{1}+\sum_{p=1}^{n}\left\|u_{1}^{p}-w_{1 h}^{p}\right\|_{1} .
$$

Consequently,

$$
\left\|u_{1}^{n+1}-u_{1 h}^{n+1}\right\|_{1} \leq \sum_{p=1}^{n+1}\left\|u_{1}^{p}-w_{1 h}^{p}\right\|_{1}+\sum_{p=0}^{n}\left\|u_{2}^{p}-w_{2 h}^{p}\right\|_{2} .
$$

For the second inequality, $i=2$, we have

$$
\begin{aligned}
\left\|u_{2}^{n+1}-u_{2 h}^{n+1}\right\|_{2} & \leq\left\|u_{2}^{n+1}-w_{2 h}^{n+1}\right\|_{2}+\left\|w_{2 h}^{n+1}-u_{2 h}^{n+1}\right\|_{2} \\
& \leq\left\|u_{2}^{n+1}-w_{2 h}^{n+1}\right\|_{2}+\left|f_{2}\left(u_{2 h}^{n}\right)-f_{2}\left(u_{2 h}^{n}\right) \|_{2}+\right| \pi_{h} M u_{1}^{n+1}-\left.\pi_{h} M u_{1 h}^{n+1}\right|_{2} \\
& \leq\left\|u_{2}^{n+1}-w_{2 h}^{n+1}\right\|_{2}+\left\|M u_{1}^{n+1}-M u_{1 h}^{n+1}\right\|_{1} \\
& \leq\left\|u_{2}^{n+1}-w_{2 h}^{n+1}\right\|_{2}+\left\|u_{1}^{n+1}-u_{1 h}^{n+1}\right\|_{1} .
\end{aligned}
$$

From (5.7), we get

$$
\left\|u_{2}^{n+1}-u_{2 h}^{n+1}\right\|_{2} \leq\left\|u_{2}^{n+1}-w_{2 h}^{n+1}\right\|_{2}+\sum_{p=1}^{n+1}\left\|u_{1}^{p}-w_{1 h}^{p}\right\|_{1}+\sum_{p=0}^{n}\left\|u_{2}^{p}-w_{2 h}^{p}\right\|_{2} .
$$

Consequently,

$$
\left\|u_{2}^{n+1}-u_{2 h}^{n+1}\right\|_{2} \leq \sum_{p=0}^{n+1}\left\|u_{2}^{p}-w_{2 h}^{p}\right\|_{2}+\sum_{p=1}^{n+1}\left\|u_{1}^{p}-w_{1 h}^{p}\right\|_{1}
$$

5.2. $L^{\infty}$ error estimate. The main result is given as follows.

Theorem 5.1. Setting $h=\max \left\{h_{1}, h_{2}\right\}$, so there exists a constant $C$ independent of $h$ and $n$ such that

$$
\left\|u_{i}-u_{i h}^{n+1}\right\|_{L^{\infty}\left(\Omega_{i}\right)} \leq C h^{2}|\log h|^{3}, \quad i=1,2 .
$$


Proof. Indeed, let $K=\max \left\{k_{1}, k_{2}\right\}$, for $i=1$ we have

$$
\begin{aligned}
\left\|u_{1}-u_{1 h}^{n+1}\right\|_{L^{\infty}\left(\Omega_{1}\right)} & \leq\left\|u_{1}-u_{1}^{n+1}\right\|_{L^{\infty}\left(\Omega_{1}\right)}+\left\|u_{1}^{n+1}-u_{1 h}^{n+1}\right\|_{L^{\infty}\left(\Omega_{1}\right)} \\
& \leq\left\|u_{1}-u_{1}^{n+1}\right\|_{L^{\infty}\left(\Omega_{1}\right)}+\sum_{p=1}^{n+1}\left\|u_{1}^{p}-w_{1 h}^{p}\right\|_{1}+\sum_{p=0}^{n+1}\left\|u_{2}^{p}-w_{2 h}^{p}\right\|_{2} \\
& \leq K^{2 n}\left\|u^{0}-u\right\|_{L^{\infty}\left(\Gamma_{1}\right)}+2(n+1) C_{1} h^{2}|\log h|^{2},
\end{aligned}
$$

where we used Lemma 4.1 and Theorem 3.1, respectively. Now, setting $K^{2 n} \leq h^{2}$ we get $\left\|u_{1}-u_{1 h}^{n+1}\right\|_{L^{\infty}\left(\Omega_{1}\right)} \leq C h^{2}|\log h|^{3}$. Similarly, we obtain the same result for $i=2$.

Remark 5.1. Confirmation for what we mentioned previously that this result is a generalization to the previous works, we note that:

(a) if the second member and the obstacle are not related to the solution, we get $[2]$;

(b) if only the obstacle is related to the solution, we get [3];

(c) if only the second member is related to the solution, we get $[4,10,11]$.

\section{REFERENCES}

[1] M. Boulbrachene, Optimal $L^{\infty}$-error estimate for variational inequalities with nonlinear source termms, Appl. Math. Lett. 15 (2002), 1013-1017.

[2] M. Boulbrachene and S. Saadi, Maximum norm analysis of an over lapping non matching grids method for the obstacl problem, Adv. Difference Equ. 2006 (2006), Paper ID 085807.

[3] M. Haiour and S. Boulaaras, Overlapping domain decomposition method for elliptic quasivarational inqualities related to impulse control problem with mixed boundary conditions, Pro. Math. Sci. 121(4) (2011), 481-493.

[4] M. Haiour and E. Hadidi, Uniform convergence of Schwarz method for varational inqualities for noncoercive variationam inequalities, International Journal of Contemporary Mathematical Sciences 4(28) (2009), 1423-1434.

[5] J. Hannouzet and P. Joly, Convergence uniform des itérés définissant la solution d'une inéquation quasi-variantionnelle, C. R. Acad. Sci. Paris, Serie A 286 (1978).

[6] P. L. Lions and P. Perthame, Une remarque sur les opérateurs nonlinéaire intervenant dand les inéquations quasi-variational, Ann. Fac. Sci. Toulouse Math. 5 (1983), 259-263.

[7] P. L. Lions, On the Schwarz alternating method I, in: R. Gowinski, G. H. Golub, G. A. Meurant and J. Péeriaux (Eds.) Proceedings of First International Symposium on Domain Decomposition Methods for Partial Differential Equations, SIAM, Philadelphia, 1988, 1-42.

[8] P. L. Lions, On the Schwarz alternating method II, stochastic interpretation and order proprieties, domain decomposition methods, in: Proceedings of Second International Symposium on Domain Decomposition Methods for Partial Differential Equations, SIAM, Philadelphia, 1989, 47-70.

[9] P. A. Raviart, J. M. Thomas, Introduction á lAnalyse Numérique des Équations aux Dérivées Partielles, $3^{\text {eme }}$ tirage, Masson, Paris, New York, Barcelone, 1992.

[10] H. Mechri and S. Saadi, Overlapping nonmateching grid method for the ergodic control quasivarational inequalities, American Journal of Computational Mathematics 3 (2013), 27-31.

[11] S. Saadi and A. Mehri, $L^{\infty}$-error estimate of Schwarz algorithm for noncoercive variational inequalities, Appl. Math. Appl. 5(3) (2014), 572-580.

[12] J. Zeng and S. Zhou, Schwarz altgorithm of the solution of variational inequalities with nonlinear source terms, Appl. Math. Comput. (1988), 23-35. 


\author{
${ }^{1}$ Faculty of Exact Sciences, Department of Mathematics, \\ UNIVERSITY ECHAHID HAMMA LAKHDAR, \\ El oued 39000, Algeria \\ Email address: beggasmr@yahoo.fr \\ ${ }^{2}$ Department of Mathematics, \\ LABORATORY LANOS, UNIVERSiTy OF ANNABA, \\ P.O.Box.12 Annaba 23000, Algeria. \\ Email address: haiourm@yahoo.fr
}

